\title{
Measles outbreak in the Philippines: epidemiological and clinical characteristics of hospitalized children, 2016-2019
}

\author{
Fleurette M. Domai, ${ }^{a}$ Kristal An Agrupis, ${ }^{b}$ Su Myat Han, ${ }^{a}$ Ana Ria Sayo, ${ }^{c}$ Janine S. Ramirez, ${ }^{c}$ Raphael Nepomuceno, ${ }^{a}$ \\ Shuichi Suzuki, ${ }^{a, b}$ Annavi Marie G Villanueva, ${ }^{a, c}$ Eumelia P. Salva, ${ }^{c}$ Jose Benito Villarama, ${ }^{c}$ Koya Ariyoshi, ${ }^{d}$ Kim Mulholland, ${ }^{a, e, f, g}$ \\ Luigi Palla, ${ }^{a, h}$ Kensuke Takahashi, ${ }^{a, d, i}$ Chris Smith, ${ }^{a, d j *}$ and Edna Miranda, ${ }^{c}$ \\ ${ }^{a}$ School of Tropical Medicine and Global Health, Nagasaki University, Japan, 852-8523 \\ ${ }^{b}$ San Lazaro Hospital-Nagasaki University Collaborative Research Office, Manila, Philippines \\ 'San Lazaro Hospital, Manila, Philippines \\ ${ }^{\mathrm{d}}$ Institute of Tropical Medicine, Nagasaki University, Nagasaki, Japan \\ 'Murdoch Children's Research Institute, Royal Children's Hospital, Melbourne, VIC 3051, Australia \\ ${ }^{f}$ Department of Paediatrics, University of Melbourne, Melbourne, VIC, Australia \\ ${ }^{9}$ Department of Infectious Disease Epidemiology, London School of Hygiene \& Tropical Medicine, London, UK \\ ${ }^{\mathrm{h}}$ Department of Public Health and Infectious Diseases, University of Rome La Sapienza, Rome, Italy \\ 'Acute \& Critical Care Unit, Nagasaki University Hospital, Nagasaki, Japan \\ jDepartment of Clinical Research, London School of Hygiene and Tropical Medicine
}

\section{Summary}

Background Measles outbreaks increased worldwide during 2017-I9. The largest outbreak in the World Health Organisation Western Pacific region occurred in the Philippines where first-dose measles-containing vaccine (MCVI) coverage had reduced to $75 \%$ in 2018 . The aim of this study was to summarise paediatric measles admissions to the national infectious diseases referral hospital in Manila during 2016 to 2019.

Methods A retrospective single-centre observational study including 5,562 children aged under five years admitted with measles from January 2016 to December 20I9. We summarised sociodemographic and clinical characteristics, vaccine status, reported exposures, and outcomes. Univariable and multivariable logistic regression analyses were undertaken to assess associations between different characteristics of hospitalised children and death.

Findings The median age of children hospitalised with measles was II months (interquartile range: $7-28$ ). $84 \cdot 5 \%$ of cases were reported not to have received any MCV. The risk of mortality was $3 \cdot 2 \%$, with $4 \mathrm{I} \%$ of deaths occurring among children aged less than 9 months. No children died who had received two MCV. The following characteristics were significantly associated with mortality in the multivariable analysis: age group, residence outside of the national capital region, not having received any MCV, duration between onset of fever and hospital admission of 7-I4 days compared with o-3 days, not receiving vitamin A supplementation, having pneumonia, and gastroenteritis.

Interpretation The Philippines remains at risk of future measles epidemics. Routine immunization needs to be strengthened and earlier timing of MCVI requires further evaluation to reduce measles incidence and mortality.

Copyright (c) 202I The Author(s). Published by Elsevier Ltd. This is an open access article under the CC BY-NC-ND license (http://creativecommons.org/licenses/by-nc-nd/4.०/)

Keywords: measles; vaccine-preventable disease; Philippines, Western-Pacific region; immunisation; paediatric infectious diseases

\section{Introduction}

Measles is a highly contagious viral disease that has been responsible for the death or disability of millions

*Corresponding author: Chris Smith, School of Tropical Medicine and Global Health (TMGH), Nagasaki University, I-I2-4 Sakamoto, 852-8523, Japan, Phone: +8I (0)80-8710-5309

E-mail address: christopher.smith@1shtm.ac.uk (C. Smith). of children. ${ }^{\mathrm{I}, 2}$ Measles incidence and mortality has declined over the past century due to improved nutrition, socioeconomic status, healthcare, and the introduction of a safe, cost-effective vaccine in the ig6o's. Annual incidence declined from around 4 million in I98I to less than 150,000 in 2016 leading to renewed interest in global eradication. ${ }^{3}$ However, global measles immunisation coverage has been stagnant for a decade
The Lancet Regional Health - Western Pacific 2021;19: 100334 Published online $\mathrm{xxx}$ https://doi.org/10.1016/j. lanwpc.2021.100334 


\section{Research in context}

Evidence before this study

Measles outbreaks increased worldwide through 201719. The largest outbreak in the World Health Organisation (WHO) Western Pacific Region occurred in the Philippines during 2018-19. The number of confirmed and compatible measles cases, deaths, and vaccination coverage in the Philippines has been reported by the WHO, and a situation analysis of the outbreak by the International Federation of Red Cross and Red Crescent Societies. We searched and PubMed for articles describing the 2018-19 measles outbreak in the Philippines published from 2017-2021, using the search terms "Measles" and "Philippines" (most recent search conducted on 27 August, 2021). Of the 31 articles, none analysed sociodemographic and clinical data of individuals with measles during the 2018-19 outbreak in the Philippines.

\section{Added value of this study}

We summarised sociodemographic and clinical characteristics amongst more than 5,000 children hospitalised with measles during 2016-19 in an infectious diseases hospital in Manila. We were able to describe measles cases according to timing of admission, age, sex, residence, reported place of exposure, vaccination status, reported barriers to non-vaccination, timing of onset of fever and rash, symptoms, complications, Vitamin A treatment, and outcome. We analysed associations with death. Thus this analysis was more detailed than what could have been undertaken using existing published data.

\section{Implications of all the available evidence}

To our knowledge, this analysis includes the largest number of individuals with measles in the Philippines ( $7.7 \%$ of the 72,496 reported cases in the Philippines during 2016-19). This was a significant measles outbreak that resulted in the unnecessary deaths of 178 children in this hospital alone (risk of mortality $3.2 \%$, compared with $2.6 \%$ reported nationally in 2019 ). Our analysis highlights the importance of Vitamin A in the treatment of measles.

In our analysis, no deaths occurred in children who were fully vaccinated. The WHO reported that coverage of the first dose of measles-containing vaccine (MCV1) in the Philippines declined to $72 \%$ in 2020 . Routine immunization needs to be strengthened to prevent further outbreaks. Further research should be undertaken to identify the health system and cultural factors associated with vaccination hesitancy in the Philippines.

Our analysis showed that $41 \%$ of deaths occurred in children aged less than nine months; with $23 \%$ occurring between 6 and 9 months. MCV1 first dose is not routinely given before nine months. We draw attention to one of the few previous trials of earlier vaccination and recommend that the measles vaccine schedule in low- and middle-income countries where maternal antibody levels may be low is re-evaluated. at around $86 \%$ for first-dose Measles Containing Vaccine (MCVI) and less than $70 \%$ for MCV2, below the 95\% recommended by the World Health Organization (WHO) to achieve herd immunity and insufficient to prevent outbreaks. ${ }^{4}$

Epidemic cycles of 2-5 years result from accumulation of susceptible individuals over successive birth cohorts and subsequent decline following outbreaks. Irregular and large outbreaks can occur in the tropics and regions with high birth rates. ${ }^{3}$ Through 20I7-I9, measles outbreaks increased worldwide, culminating in more than 800,000 cases being reported to the WHO in 20I9, the majority in Africa associated with large outbreaks in Madagascar and the Democratic Republic of the Congo. ${ }^{5}$

The largest outbreak in the WHO Western Pacific region occurred in the Philippines, with reported cases increasing from 2,428 in 2017 to 20,827 in 2018 , and 48,525 in 2019. ${ }^{6}$ Outbreak clusters were first reported in the southern Island of Mindanao in September 2017, followed by the National Capital Region (NCR). A measles outbreak was declared in five regions, including the NCR, in February 20I8. ' In the following weeks, outbreaks reached I7 regions. Supplemental Immunisation Activity (SIA) campaigns were conducted in NCR and Mindanao (Phase I) and in the Visayas and Luzon (Phase 2) during 20I8, and in all regions during 2019, targeting children aged 6-59 months as well as older children. At the peak of the epidemic, measles care units were established to support overcrowded hospitals. Reported cases declined over several months to pre-epidemic levels by July 2019. Pockets of measles outbreaks were reported and 3,832 cases in 2020, with preliminary reports of $\mathrm{I} 23$ cases during January to June, 2021. 8

Measles vaccination coverage in the Philippines was in decline prior to the 20I8-I9 outbreak. The measles vaccine was introduced in the Philippines in I983, with a second dose added to the schedule in 2009. MCVI is offered to children at 9 months and $\mathrm{MCV}_{2}$ at $\mathrm{I} 2-\mathrm{I} 5$ months, combined mumps, and rubella. ${ }^{9}$ MCVI coverage peaked at $92 \%$ from 2004 to 2008 but gradually declined over the following decade to $80 \%$ in 2016 and $75 \%$ in $2018 .^{\text {IO }}$ Measles outbreaks occurred in 2010-II and 20I3. 9,II The 'Dengvaxia' controversy in late 2017 has been cited as a contributing factor to the further decline of MCVI coverage in 20I8. ${ }^{\text {I2 }}$

In this study we analyse epidemiological and clinical data from San Lazaro Hospital (SLH), the national infectious diseases referral hospital serving a densely populated low-income population in Manila, the Philippines where more than 5,000 children were hospitalised with measles from I January 2016 to 3I December 20I9. The aim of this study was to summarise the outbreak in Manila, and consider implications for the Philippines and the wider region. 


\section{Methods}

\section{Study design and participants}

We conducted a retrospective single-centre observational study including all children aged under five years admitted with measles at SLH from January 2016 to December 20I9. During this time, individuals with suspected measles were admitted to SLH, in addition to other hospitals in the region. Admitted children included either self-referrals (walk-in patients) or referrals from other health facilities.

The study was approved by the SLH research ethics and review unit (Ref: SLH-RERU-2020-OIO-E Ver 2) and the School of Tropical Medicine and Global Health, Nagasaki University ethical committee (Ref: NU_TMGH_2020-106-0). This was a retrospective analysis of routinely collected anonymised data thus patient or caregiver consent was not required.

\section{Procedures}

We obtained data from Philippine Integrated Disease Surveillance and Response Case Investigation Forms (CIF) completed by the clinical teams submitted to the SLH Epidemiology department. CIF forms were completed for individuals fulfilling the clinical case definition for suspected measles, namely a fever $(3 \overline{8} \mathrm{C}$ or more), maculopapular rash (non-vesicular), and at least one of either cough, coryza or conjunctivitis, as per the WHO-recommended surveillance standard. ${ }^{\mathrm{I3}}$ The CIF collected information on the date of admission, measles vaccination status according to the report of the caregiver or the vaccination card (if available), barriers to vaccination and likely place of exposure according to the caregiver report, socio-demographic, clinical characteristics, whether Vitamin A was given during the current illness, and outcome. Data was not available on characteristics of the rash, nutrition status, routine Vitamin A supplementation, the overall vaccine status of cases, or whether the case was a likely primary or secondary household case. Selected data were extracted to create an anonymised dataset.

\section{Statistical analysis}

Univariable and multivariable Firth (penalised likelihood) logistic regression analyses were undertaken to assess associations between different characteristics of hospitalised children and death (outcome). Odds ratios (ORs) were calculated with a $95 \%$ confidence interval (CI), and a p-value $<0.05$ was considered statistically significant. Additionally, a matrix summarising the association between all pairs of variables was produced and this, combined with prior knowledge of substantive science, informed the selection of variables to include in the model. Age group, sex (as known confounders), at least one dose of MCV, residency of Manila, Vitamin A supplementation and duration of fever before admission (as significantly associated to mortality) were initially included in the model to assess the background risk factors for morbidity. Clinical diagnosis of pneumonia, neurological symptoms and gastroenteritis were included in a second more comprehensive model (see Supplementary Material) after checking that their association with other model covariates would not confound the other variables' relationship with mortality. Endemic season was removed from both multivariable models as identified to be on the causal pathway from vaccination to mortality. Although the number of univariable tests was large, we did not formally apply a correction for multiple testing because the variable selection strategy for the multivariable model was only partly based on p-values. For more detail of selection of variables, please refer to the Supplementary Material. Stata IC I6.I was used for the main analyses. ${ }^{\text {I4 }}$ QGIS software was used for mapping and distance calculation (QGIS 3.I4). ${ }^{15}$

\section{Role of the funding source}

The funder of the study had no role in the study design, data collection, data analysis, data interpretation, or the writing of the report. The corresponding author had full access to all the data in the study and had final responsibility for the decision to submit for publication.

\section{Results}

5,562 children aged under 5 years fulfilling a clinical case definition for measles admitted to SLH between I Jan 2016 and 3I Dec 20I9 were included in this study.

Figure I shows measles cases by month of rash onset in the WHO Western Pacific Region, numbers of children admitted to SLH per week, and the approximate residence of hospitalised children during the study period. The 20I8-19 epidemic in the Western Pacific region peaked in February 2019, dominated by measles cases in the Philippines. This corresponded to the trend of admissions to SLH, with low levels of admissions through 20I6-I7, increasing numbers through 20I8, peaking at over 300 cases per week during two weeks in February 2019, then decreasing to pre-epidemic levels by July 20I9. Trends of increasing cases being admitted to SLH from outside of the National Capital Region (NCR) can be observed during 2018 and 2019 .

Table I describes the demographic and clinical characteristics of the 5,562 children hospitalised with measles. The majority of admissions $(5,206$ [94\%]) were during the period of the epidemic between February 2018 and July 20I9. Just over half were male $(55 \%)$. The median age was II months (interquartile range [IQR]: 7 to 28 ); $51 \%$ were aged less than one year, $19 \%$ aged I2 years, I2\% $2-3$ years, and I7\% aged more than three years. The majority of hospitalised children $(88 \%)$ were 


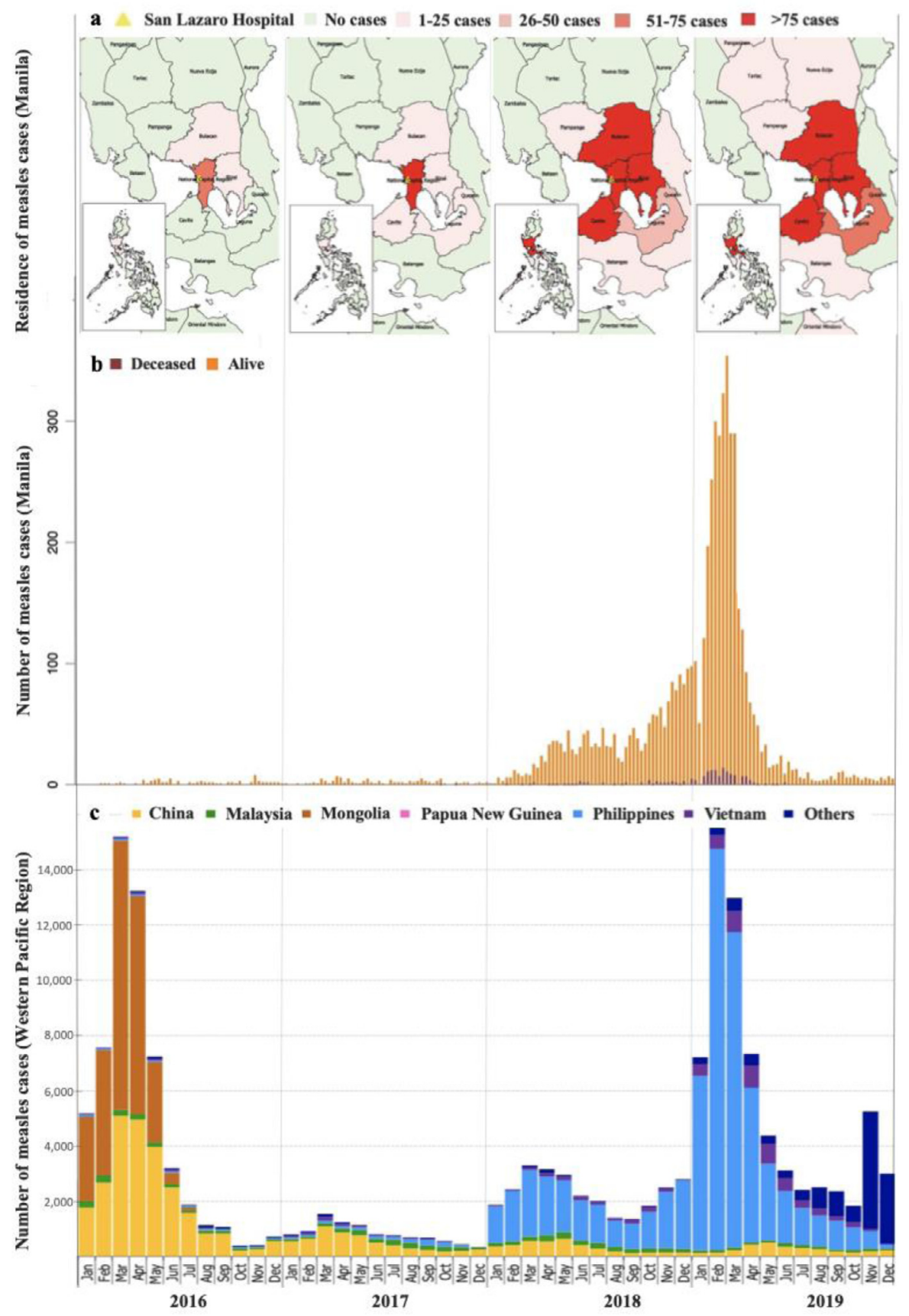

Figure 1. Trends in measles cases in the Western Pacific Region, San Lazaro Hospital (SLH), Manila, the Philippines, 20162019 (a) Distribution of hospitalized measles cases admitted to SLH by location (b) Epidemic curve of the hospitalized measles cases at SLH Manila by week of admission, and (c) Time-series of reported confirmed and compatible measles cases in the Western Pacific Region by month of rash onset, 2016-2019 (adapted from: WHO Measles-Rubella Bulletin ${ }^{8}$ )

from the NCR and lived within Io kilometres of the hospital.

The majority of children $(84.5 \%)$ were reported not to have received any measles vaccination, $6 \cdot 7 \%$ one dose (MCVI), O. $15 \%$ two doses $\left(\mathrm{MCV}_{2}\right)$, with vaccination but number of doses unknown in $8.7 \%$. Amongst children aged over nine months, $22 \%$ had received one or more measles vaccination. Aside from children who were reported to have been age-ineligible, many reasons were cited as barriers to vaccination, the most common being 'child was sick' (I7\%) and 'mother/caregiver was unavailable' (I7\%). Place of residence was the most 


\begin{tabular}{|c|c|}
\hline Characteristics $(\mathrm{N}=5,562)$ & $\mathbf{N}(\%)$ \\
\hline \multicolumn{2}{|l|}{ Year of admission } \\
\hline 2016 & $87(1 \cdot 6 \%)$ \\
\hline 2017 & $116(2 \cdot 1 \%)$ \\
\hline 2018 & $2,062(37 \cdot 1 \%)$ \\
\hline 2019 & $3,297(59 \cdot 3 \%)$ \\
\hline \multicolumn{2}{|l|}{ Admission timing } \\
\hline Non-epidemic & $356(6.4 \%)$ \\
\hline Epidemic (Feb 2018-July 2019) & $5,206(93 \cdot 6 \%)$ \\
\hline \multicolumn{2}{|l|}{ Sex } \\
\hline Male & $3,062(55 \%)$ \\
\hline Female & $2,500(45 \%)$ \\
\hline Age in months (median, IQR) & $11(7-28)$ \\
\hline \multicolumn{2}{|l|}{ Age group, in months } \\
\hline$<3$ & $94(1.7 \%)$ \\
\hline 3-5 & $715(12 \cdot 9 \%)$ \\
\hline $6-8$ & $1,280(23 \%)$ \\
\hline $9-11$ & $765(13 \cdot 8 \%)$ \\
\hline $12-23$ & $1,066(19 \cdot 1 \%)$ \\
\hline $24-35$ & $670(12 \cdot 1 \%)$ \\
\hline $36-47$ & $564(10 \cdot 1 \%)$ \\
\hline $48-59$ & $408(7 \cdot 3 \%)$ \\
\hline \multicolumn{2}{|l|}{ Region of residence ( $N=5,376$ ) } \\
\hline NCR & $4,706(87 \cdot 5 \%)$ \\
\hline Calabarzon & $669(12 \cdot 4 \%)$ \\
\hline Mimaropa & $1(0 \cdot 0 \%)$ \\
\hline \multicolumn{2}{|l|}{ Distance from the hospital (km) } \\
\hline$<5$ & $2,423(43.6 \%)$ \\
\hline $5-10$ & $1,244(22 \cdot 4 \%)$ \\
\hline $11-20$ & $1,283(23.1 \%)$ \\
\hline$\geq 21$ & $612(11.0 \%)$ \\
\hline \multicolumn{2}{|c|}{ Place of exposure according to caregiver report } \\
\hline Residence & $3,142(58 \cdot 8 \%)$ \\
\hline Health care facility & $240(4 \cdot 5 \%)$ \\
\hline Child care facility & $17(0 \cdot 3 \%)$ \\
\hline Other & $589(10 \cdot 0 \%)$ \\
\hline Unknown & $1,357(25.4 \%)$ \\
\hline \multicolumn{2}{|l|}{ Vaccination status (all age, $\mathrm{N}=\mathbf{5 , 4 4 7}$ ) } \\
\hline Not vaccinated & $4,600(84 \cdot 5 \%)$ \\
\hline Vaccinated 1 dose & $364(6 \cdot 7 \%)$ \\
\hline Vaccinated 2 doses & $8(0 \cdot 15 \%)$ \\
\hline Vaccinated (number of doses unknown) & $475(8 \cdot 7 \%)$ \\
\hline \multicolumn{2}{|c|}{ Vaccine status (aged over 9 months, $\mathrm{N}=3,372$ ) } \\
\hline Not vaccinated & $2,643(78.4 \%)$ \\
\hline Vaccinated ( $\geq 1$ dose) & $729(21.6 \%)$ \\
\hline \multicolumn{2}{|c|}{ Age at time of first measles vaccination $(N=847)$} \\
\hline$<9$ months & $67(7.9 \%)$ \\
\hline$>9$ months & $143(16.9 \%)$ \\
\hline Unknown & $637(75.2 \%)$ \\
\hline \multicolumn{2}{|c|}{ Reported barriers to vaccination ( $N=4,739$ ) } \\
\hline Age-ineligible & $1,810(38 \cdot 2 \%)$ \\
\hline No vaccine available & $58(1 \cdot 2 \%)$ \\
\hline No vaccinator present & $15(0 \cdot 3 \%)$ \\
\hline Medical contraindication & $6(0 \cdot 1 \%)$ \\
\hline Child was sick & 827 (17.4\%) \\
\hline
\end{tabular}

\begin{tabular}{|c|c|}
\hline Characteristics $(\mathrm{N}=5,562)$ & $\mathbf{N}(\%)$ \\
\hline Moved residence & $230(4 \cdot 8 \%)$ \\
\hline Mother/caregiver was unavailable & $814(17 \cdot 2 \%)$ \\
\hline Against belief & $20(0.4 \%)$ \\
\hline Fear of side effects & $73(1.5 \%)$ \\
\hline Missed schedule & $156(3 \cdot 3 \%)$ \\
\hline Other reasons ${ }^{\ddagger}$ & $82(1.7 \%)$ \\
\hline Dengvaxia scare & $25(0.5 \%)$ \\
\hline Difficult access to health care centre & $21(0.4 \%)$ \\
\hline Lost vaccination record & $32(0 \cdot 7 \%)$ \\
\hline Uninformed/unaware & $4(0 \cdot 1 \%)$ \\
\hline Unrecalled/unknown & $648(13 \cdot 7 \%)$ \\
\hline \multicolumn{2}{|l|}{ Clinical information } \\
\hline Cough & $5,469(98 \cdot 3 \%)$ \\
\hline Coryza & $4,489(80 \cdot 7 \%)$ \\
\hline Conjunctivitis & $2,583(46 \cdot 5 \%)$ \\
\hline Koplik's spots & $1,339(25 \cdot 8 \%)$ \\
\hline Pneumonia & $3,764(67 \cdot 7 \%)$ \\
\hline Neurological complications & $15(0 \cdot 3 \%)$ \\
\hline Gastroenteritis & $209(3 \cdot 8 \%)$ \\
\hline Upper respiratory tract infection & $4(0 \cdot 1 \%)$ \\
\hline Dehydration & $3(0 \cdot 1 \%)$ \\
\hline \multicolumn{2}{|c|}{ Duration between fever onset and admission (days) } \\
\hline $0-3$ & $2,071(37 \cdot 5 \%)$ \\
\hline $4-6$ & $2,746(49.7 \%)$ \\
\hline $7-14$ & $676(12 \cdot 2 \%)$ \\
\hline$>14$ & $35(0.63 \%)$ \\
\hline \multicolumn{2}{|c|}{ Duration between rash onset and admission (days) } \\
\hline $0-3$ & $4,757(87 \%)$ \\
\hline $4-6$ & $580(10 \cdot 6 \%)$ \\
\hline $7-14$ & $112(2 \cdot 1 \%)$ \\
\hline$>14$ & $16(0 \cdot 3 \%)$ \\
\hline \multicolumn{2}{|c|}{ Vitamin A supplementation $(\mathrm{N}=5,037)$} \\
\hline Received during current illness & $4,269(84 \cdot 8 \%)$ \\
\hline \multicolumn{2}{|l|}{ Outcome } \\
\hline Discharged & $5,384(96 \cdot 8 \%)$ \\
\hline Died & $178(3 \cdot 2 \%)$ \\
\hline \multicolumn{2}{|c|}{$\begin{array}{l}\text { Table 1: Demographic and clinical characteristics of children } \\
\text { hospitalised with measles }\end{array}$} \\
\hline \multicolumn{2}{|c|}{$\begin{array}{l}\text { Data are } \mathrm{n}(\%) \text { or median (IQR). Subcategory totals do not add up to } 5,562 \\
\text { where there was missing data. } \\
\ddagger \text { No further information was available on data recorded as 'other' on } \\
\text { the case investigation form. } \\
\text { \& Data were not available on pathogens causing pneumonia or } \\
\text { gastroenteritis. }\end{array}$} \\
\hline
\end{tabular}

commonly reported site of probable exposure to measles (59\%).

Classical symptoms of cough, coryza, and conjunctivitis (the " 3 Cs") were reported in most cases: $98 \%$ for cough, $8 \mathrm{r} \%$ for coryza, and $47 \%$ for conjunctivitis, respectively. Koplik's spots were reported in $26 \%$ of cases. The time between onset of fever and rash to hospital admission were reported to be less than one week in $87 \%$ and $98 \%$, respectively. Complications were 


\begin{tabular}{|c|c|c|c|c|c|c|}
\hline & Total $\mathbf{n}$ & Died n (\%) & Crude OR & P-value & Adjusted OR* & P-value \\
\hline \multicolumn{7}{|l|}{ Age group (months) } \\
\hline$<3$ & 94 & $2(2.1)$ & $1.07(0.29-3.90)$ & 0.919 & $1.23(0.33-4.65)$ & 0.758 \\
\hline $3-5$ & 715 & $31(4.3)$ & $1.82(1.13-2.93)$ & 0.013 & $1.68(0.99-2.85)$ & 0.055 \\
\hline $6-8$ & 1,280 & $40(3.1)$ & $1.29(0.83-2.01)$ & 0.256 & $1.34(0.82-2.17)$ & 0.243 \\
\hline $9-11$ & 765 & $21(2.7)$ & $1.14(0.67-1.94)$ & 0.622 & $1.13(0.62-2.04)$ & 0.695 \\
\hline $12-24$ & 1,066 & $44(4.1)$ & $1.72(1.12-2.66)$ & 0.014 & $1.49(0.91-2.44)$ & 0.116 \\
\hline \multirow[t]{2}{*}{$>24$} & 1,642 & $40(2.4)$ & Ref & & Ref & \\
\hline & & & & 0.890 & & 0.004 \\
\hline \multicolumn{7}{|l|}{ Sex } \\
\hline Male & 3,062 & $100(3.3)$ & Ref & & Ref & \\
\hline Female & 2,500 & $78(3.1)$ & $0.96(0.71-1.29)$ & 0.765 & $0.95(0.68-1.32)$ & 0.738 \\
\hline \multicolumn{7}{|l|}{ Region of residence } \\
\hline In NCR & 4,706 & $140(3)$ & Ref & & Ref & \\
\hline Outside NCR & 670 & $30(4.5)$ & $1.55(1.04-2.31)$ & 0.032 & $1.61(1.05-2.48)$ & 0.030 \\
\hline \multicolumn{7}{|l|}{ Admission timing } \\
\hline Non-epidemic & 356 & $3(0.8)$ & Ref & & & \\
\hline Epidemic & 5,206 & $175(3.4)$ & $3.52(1.22-10.20)$ & 0.020 & $\ldots$ & \\
\hline \multicolumn{7}{|l|}{ Vaccine status } \\
\hline Vaccinated ( $\geq 1$ dose) & 847 & $16(1.9) \dagger$ & Ref & & Ref & \\
\hline Non-vaccinated & 4,600 & $154(3.3)$ & $1.75(1.05-2.93)$ & 0.032 & $1.86(1.04-3.33)$ & 0.036 \\
\hline \multicolumn{7}{|c|}{ Duration between fever onset and admission (days) } \\
\hline $0-3 d$ & 2,071 & $48(239)$ & Ref & & Ref & \\
\hline $4-6 d$ & 2,746 & $91(3.3)$ & $1.44(1.01-2.05)$ & 0.044 & $1.46(0.99-2.16)$ & 0.058 \\
\hline $7-14 d$ & 676 & $37(5.5)$ & $2.45(1.58-3.78)$ & $<0.001$ & $2.38(1.47-3.86)$ & $<0.001$ \\
\hline \multirow[t]{2}{*}{$>14 d$} & 35 & $1(2.9)$ & $1.81(0.35-9.53)$ & 0.482 & $0.64(0.04-10.69)$ & 0.753 \\
\hline & & & & 0.001 & & $<0.001$ \\
\hline \multicolumn{7}{|c|}{ Duration between rash onset and admission (days) } \\
\hline $0-3 d$ & 4,757 & $136(2.9)$ & Ref & & & \\
\hline $4-6 d$ & 580 & $30(5.2)$ & $1.88(1.25-2.81)$ & 0.002 & $\ldots$ & \\
\hline $7-14 d$ & 112 & $11(9.8)$ & $3.84(2.04-7.23)$ & $<0.001$ & $\ldots$ & \\
\hline \multirow[t]{2}{*}{$>14 d$} & 16 & $0(0)$ & $1.03(0.06-17.19)$ & 0.986 & $\ldots$ & \\
\hline & & & & $<0.001$ & $\ldots$ & \\
\hline \multicolumn{7}{|c|}{ Vitamin A supplementation } \\
\hline Not received & 768 & $41(5.3)$ & $1.96(1.37-2.82)$ & $<0.001$ & $1.93(1.31-2.85)$ & 0.001 \\
\hline \multicolumn{7}{|l|}{ Clinical information } \\
\hline Cough & 5,469 & $178(3.3)$ & $6.31(0.39-102.03)$ & 0.195 & $\ldots$ & \\
\hline Coryza & 4,489 & $141(3.1)$ & $0.90(0.62-1.30)$ & 0.571 & $\ldots$ & \\
\hline Conjunctivitis & 2,583 & $77(3)$ & $0.88(0.65-1.18)$ & 0.389 & $\ldots$ & \\
\hline Koplik's spot & 1,339 & $37(2.8)$ & $0.80(0.55-1.16)$ & 0.234 & $\ldots$ & \\
\hline Pneumonia & 3,764 & $147(3.9)$ & $2.29(1.55-3.38)$ & $<0.001$ & $2.40(1.56-3.69)$ & $<0.001$ \\
\hline Neurological & 15 & $2(13.3)$ & $5.64(1.45-21.91)$ & 0.013 & $1.79(0.27-12.04)$ & 0.549 \\
\hline Gastroenteritis ${ }^{\pi}$ & 209 & $15(7.2)$ & $2.53(1.47-4.34)$ & 0.001 & $3.61(2.00-6.51)$ & $<0.001$ \\
\hline \multicolumn{7}{|c|}{ Table 2: Associations with mortality } \\
\hline \multicolumn{7}{|c|}{$\mathrm{OR}=$ odds ratio. } \\
\hline \multicolumn{7}{|c|}{ Individual and overall p-values are presented for categorical variables } \\
\hline
\end{tabular}

reported in the majority of children, most commonly pneumonia (68\%) and gastroenteritis (4\%). Neurological complications were reported in $\mathrm{I}_{5}(0.3 \%)$ children. $85 \%$ of children received Vitamin A during their current illness. I78 (3.2\%) children died and 5,384 (96.8\%) were discharged.
Table 2 shows associations between socio-demographic and clinical characteristics and death. The following characteristics were significantly associated with mortality in the univariable analysis: residence outside of the NCR, admission during the period of the epidemic, not having received any MCV, not receiving 
vitamin A supplementation, duration between onset of fever or duration between onset of rash and hospital admission, pneumonia, neurological complications, and gastroenteritis. No child died who had received two doses of MCV. Children with the following risk factors had significantly higher odds of mortality in the multivariable analysis: residence outside of the NCR (adjusted OR [AOR] I.6I, 95\% CI I.05-2.48), not having received any MCV (AOR I.86, 95\% CI I. 04-3.33), duration between onset of fever and hospital admission of 7I4 days compared with 0-3 days (AOR 2.38, 95\% CI $\mathrm{I} \cdot 47-3.86$ ), not receiving vitamin A supplementation (AOR I.93, 95\% CI I.3I-2.85), pneumonia (AOR 2.40, 95\% CI I.56-3.69), and gastroenteritis (AOR 3.6I, 95\% CI $2 \cdot 00-6 \cdot 5 \mathrm{I})$. The association between age group and mortality had overall significance $(\mathrm{p}=0.004$ by likelihood ratio test), but not within an individual category. The association between neurological complications in the univariable analysis did not remain statistically significant in the multivariable analysis. Sex, or the presence of cough, coryza, conjunctivitis, or Koplik's spots were not significantly associated with mortality in the uni- or multivariable analyses.

\section{Discussion}

In this study we describe the epidemiological and clinical characteristics of more than 5,000 children hospitalized with measles in Manila, the majority during the 20I8-I9 epidemic in the Philippines. To our knowledge, this analysis includes the largest number of individuals affected by the epidemic in the Philippines, representing around $7 \cdot 7 \%$ of the 72,496 reported measles cases in the Philippines during 2016-19. ${ }^{6}$ The epidemic curve of admitted cases in our study has a similar pattern to that of the country and western pacific region, suggesting that our study population is temporally representative of the wider epidemic. The majority of hospitalised children lived within Io $\mathrm{km}$ of the hospital, situated in the high-density urban slum area of Tondo. Eighty-five percent $(85 \%)$ had not received any MCV according to the caregiver report or immunisation card. More than half of cases in our study reported residence as the likely place of infection exposure, suggesting secondary cases. Crowded conditions and high population densities are known risk factors for the disease's occurrence and spread. ${ }^{3,16}$ NCR is the densest region in the Philippines with a population of 20,785 per square kilometre, ${ }^{17}$ and has been repeatedly cited as a hotspot of previous epidemics in the country. ${ }^{\mathrm{I}}$ Children living further away admitted during the epidemic likely represent referrals from other hospitals due to complications. Our study population therefore likely includes a higher proportion of severe cases than the country average.

The median age of children was under 2 years, as might be expected from an outbreak in a densely populated urban setting with high birth rates and low vaccination coverage. ${ }^{3}$ Fifty-nine percent $(59 \%)$ of 178 deaths were in children aged 9 months to 5 years. Routine immunisation needs to be strengthened in order to increase MCVI coverage and reduce cases and deaths in this age group. The strategy is less clear for the $4 \mathrm{I} \%$ deaths in children aged $<9$ months, before their scheduled MCVI. The relatively low proportion of children aged $0-<3$ months ( $2 \%$ of cases and I\% of deaths) suggests passively-acquired maternal anti-measles IgG protection. This protection is known to be higher in infants born to women with a history of wildtype measles infection compared with vaccine-induced immunity. ${ }^{\text {I9 }}$ Data on prior maternal infection or vaccination status was not available, but it is unlikely that many mothers would have received both MCVI and 2 in childhood given the relatively recent introduction of $\mathrm{MCV} 2$ in $2009 .{ }^{9}$ Thirteen percent of cases and $I 7 \%$ of deaths were in children aged between 3 and 5 months. Maternal antibodies wane during the first 3-5 months of life, leaving children susceptible to measles until receiving vaccination. The measles vaccine is not normally given to this age group due to the inhibitory effect of maternal antibodies and immaturity of the immune system, resulting in lower levels of protection. ${ }^{3}$ The optimal strategy to protect this susceptible 3-5 month age group is to reduce their exposure by achieving herd immunity in older children. Children aged between 6-8 months accounted for $23 \%$ of measles cases and 40 deaths. Some children aged between 6 and 9 months would have received measles vaccination through the SIA in 20I8. These activities need to be quick and effective in responding in the case of future outbreaks. Measles vaccination at 4.5 months of age was effective in preventing serologically confirmed and definite clinical measles during an outbreak in Guinea-Bissau in 2003-2004. ${ }^{20}$ Earlier timing of MCV in low-income settings where maternal antibody levels may be low requires further evaluation.

Children in this study presented with fever and rash and the "3 C's" symptoms were frequently reported. Pathognomonic Koplik spots were observed in $26 \%$ of children, reflecting the fleeting nature of this sign. Almost all children had a cough, reflecting the hospitalised study population. Most children had complications, with pneumonia, the most common measles complication, reported in two-thirds of cases., ${ }^{3,21,22}$ Other complications were less commonly reported. No further information was available on the pathogens causing pneumonia or gastroenteritis, or the nature of the neurological complications.

The risk of mortality amongst hospitalised children in this study was $3 \cdot 2 \%$, higher than the $2.6 \%$ measles mortality reported in the Philippines in 2019, but lower than the $5 \%$ mortality reported in endemic areas of SubSaharan Africa and Asia.,7 There was an overall significant effect of age in the multivariable analysis. Compared to the older (reference) age group, the odds of mortality were higher in the younger age groups, 
although not statistically significant, with the highest odds among children between 3-5 months which could be consistent with waning immunity. ${ }^{23}$ The odds of death were increased during the epidemic period, which may reflect more severe cases being admitted and challenges maintaining quality of care due to high numbers of admissions. There were no statistically significant differences in mortality by sex. The odds of death were increased among children residing further away from the hospital, likely reflecting referrals from other healthcare facilities due to complications. Delayed referral due to distance from the hospital likely explains the observed associations between increased duration of fever and rash and death. The odds of death were higher amongst children with complications of pneumonia or gastroenteritis, as expected and consistent with other studies. ${ }^{24,25}$ The odds of death were nearly two times higher amongst the $15 \%$ of children that did not receive Vitamin A supplementation during their current illness, highlighting the importance of this simple and low-cost intervention. ${ }^{26,27}$ The odds of death were almost two times higher amongst unvaccinated children compared with children who had received one or more doses of MCV with no deaths amongst those who had received both doses. Vaccine-modified measles in children with previous immunity following vaccination is characterised by a milder illness., ${ }^{3,2}$

Reported barriers to vaccination in our study included medical contraindication/fear of side-effects and guardian occupation. Qualitative studies are better suited to gain a deeper understanding of vaccine hesitancy in the Philippines, particularly in populations with immunity gaps where clusters have first emerged previously. The "Dengvaxia controversy" in 2017 appears to have dented vaccine confidence, ${ }^{29}$ but coverage had already been declining suggesting that a broader examination to include is required. Further research should be undertaken to identify the health system and cultural factors associated with vaccination in the Philippines to seek solutions to these problems. Ongoing efforts are required to combat anti-vaccine campaigns.

It is not a question of if, but when the next measles epidemics will occur in the Philippines and in other countries. Reported measles cases have reduced in 2020 and the first six months of $2020,{ }^{8}$ likely due to COVID-I9 social distancing measures, reduced domestic and international travel, disturbance in surveillance and reporting, and the phase of the measles epidemic cycle. However, at the same time, the COVID-I9 pandemic severely disrupted routine vaccination activities, with estimates of more than 90 million children missing measles vaccine doses by October 2020 , and reports of a lost generation of children. ${ }^{2} \mathrm{MCV}$ I coverage in the Philippines declined further to $72 \%$ in $2020 .^{\text {IO }}$ Increasing numbers of unimmunised children susceptible to measles create an environment for measles to return when COVID-I9 restrictions are relaxed. Repeated occurrences of measles outbreaks in the Philippines demonstrate the importance of re-evaluating measles virus dynamics and prevention and control strategies. ${ }^{3 \circ}$ Actions are required to reach unimmunised children through catch-up campaigns and to prepare for expected outbreaks. Governments should not lose sight of measles elimination targets as per the Measles and Rubella Strategic Framework, 202I-2030. ${ }^{3 \mathrm{I}}$

This study has some limitations. First, our study population may not be representative of measles cases in the Philippines as all individuals were hospitalised. Second, all of the children in our study were clinically diagnosed, not laboratory confirmed. It is therefore possible that some cases of diseases with similar presentations to measles such as dengue fever or rubella were included in our study sample. However, the measles case definition has high sensitivity and positive predictive value in endemic settings, and laboratory confirmation of a sub-sample of cases was being undertaken at the Research Institute for Tropical Medicine (RITM) in the Philippines. ${ }^{32}$ Datasets of laboratory confirmed cases from LMICs of the size utilised in our study are likely to be rare. Third, detailed data were lacking on the nutritional, breastfeeding and overall vaccine status of the children, number and timing of Vitamin A doses, and number of measles-containing vaccinations in some cases. The extent of recall bias affecting vaccination status data is unclear as the data source (vaccination card or caregiver report) was not recorded on the CIF. Nevertheless, due to the systematic data recording on the CIF, we were able to analyse a large population of more than 5,000 measles cases with detailed clinical information and other socio-demographic factors. Due to underreporting, measles mortality estimates are derived from models based on the number and age distribution of reported cases, measles vaccine coverage, and age and country-specific case fatality ratios. ${ }^{3}$ This is the first study showcasing the burden of measles in recent years in the Philippines. Empirical data such as presented in this study are important to inform these models.

In conclusion, we report clinical and epidemiological data from a large measles outbreak affecting mostly unvaccinated children in the Philippines with a $3 \cdot 2 \%$ case fatality rate. $4 \mathrm{I} \%$ of deaths were among children aged less than 9 months. No children died who had received two measles-containing vaccines (MCV). MCVI coverage in the Philippines declined to $72 \%$ in 2020. Routine immunization needs to be strengthened and earlier timing of MCVI requires further evaluation to prevent further outbreaks.

\section{Contributors}

Fleurette M Domai: study design, literature search, data analysis, data interpretation, writing - original draft; 
Kristal An Agrupis: data analysis, data interpretation, writing - review and editing; Su Myat Han: data analysis, Figures, writing - review and editing; Ana Ria Sayo: conceptualisation, data collection, data interpretation, writing - review and editing; Janine S. Ramirez: data collection; Raphael Neponuceno: data analysis, Figures; Shuichi Suzuki: Project administration, Data interpretation; Annavi Marie G Villanueva: data interpretation, writing - review and editing; Eumelia P. Salva: data collection, data interpretation, writing - review and editing; Jose Villarama: data interpretation, writing - review and editing; Koya Ariyoshi: study design, data interpretation; Kim Mulholland: data interpretation, writing review and editing; Luigi Palla: data analysis, writing review and editing; Kensuke Takahashi: data analysis, data interpretation, writing; Chris Smith: conceptualisation, study design, data interpretation, supervision, writing - original draft; Edna Miranda: Conceptualisation, data collection, writing - review and editing

\section{Data sharing}

The dataset for this study is available from the corresponding author and San Lazaro Hospital on reasonable request. Data without names and identifiers will be made available with a signed data access agreement after approval of a proposal by the corresponding author and the San Lazaro Hospital Epidemiology Department.

Editor note: The Lancet Group takes a neutral position with respect to territorial claims in published maps and institutional affiliations.

\section{Declaration of Competing interest}

$\mathrm{KM}$ is a member of the WHO Strategic Advisory Group of Experts (SAGE) on Immunization. The other authors declare no conflicting interests.

\section{Funding}

This work is in part funded by Nagasaki University (salary support for CS, KAA, RN, SS, KA, KT).

\section{Acknowledgments}

This work is in part funded by Nagasaki University (salary support for CS, KAA, RN, SS, KA, KT). The funder of the study had no role in the study design, data collection, data analysis, data interpretation or the writing of the report. The corresponding author had full access to all the data in the study and had final responsibility for the decision to submit for publication. We thank the San Lazaro Hospital Epidemiology department data encoders. We thank Hiroyuki Moriuchi, LayMyint Yoshida, Michiko Toizumi, and Lina Madaniyazi for comments on previous versions of the manuscript and advising on the data analysis.

\section{Supplementary materials}

Supplementary material associated with this article can be found in the online version at doi:Io.IoI $6 / j$. lanwpc.2021.100334.

\section{References}

I Cliff A, Haggett P, Smallman-Raynor M. Measles. A historical geography of a major human viral disease from global expansion to local retreat. Oxford, England: Blackwell; I993. p. I840-990.

2 Mulholland K, Kretsinger K, Wondwossen L, Crowcroft N. Action needed now to prevent further increases in measles and measles deaths in the coming years. Lancet 2020;396(I0265):I782-4

3 Moss WJ. Measles. Lancet 20I7;390(IOIII):2490-502.

4 World Health Organization. In: Global measles and rubella strategic plan: 20I2-2020. [Internet]; 20I2. Available from http://apps.who. int/iris/bitstream/Io665/44855/I/978924I503396_eng.pdf.

5 Wariri O, Nkereuwem E, Erondu NA, Edem B, Nkereuwem OO, Idoko OT, et al. A scorecard of progress towards measles elimination in I5 west African countries, 200I -I9: a retrospective, multicountry analysis of national immunisation coverage and surveillance data. Lancet Glob Heal 2020;9(3):e280-90.

6 World Health Organization. In: WHO vaccine-preventable diseases monitoring system. 2020 global summary [Internet]; 2020. [cited 202I Aug 22]. Available from https://apps.who.int/immunization_monitoring/globalsummary/countries? countrycriteria $\% 5$ Bcountry ${ }_{5} \mathrm{D} \% 5 \mathrm{~B} \% 5 \mathrm{D}=\mathrm{PHL}$.

7 International Federation of Red Cross and Red Crescent Societies. In: Operations Update Philippines: Re-emergence of Vaccine Preventable Diseases - Measles outbreak [Internet]; 2020. Available from: https://reliefweb.int/sites/reliefweb.int/files/resources/ MDRPHo32I2m_measles.pdf.

8 WHO Western Pacific Region. In: Measles-Rubella Bulletin [Internet]; 202I. Vol. I5, Measles-Rubella bulletinAvailable from https:// apps.who.int/iris/handle/I0665/339779.

9 Ylade MC. Epidemiology of measles in the Philippines. Acta Med Philipp 20I8;52(4):380-9.

Io World Health Organization. In: Measles, Ist dose (MCVI) Immunization coverage estimates by country [Internet]; 202I. [citedApr I2]. Available from: https://apps.who.int/gho/data/view.main.80ioo? lang=en.

II Ching PK, Zapanta MJ, de Los Reyes VC, Tayag E, Magpantay R. Investigation of a measles outbreak in Cordillera, Northern Philippines, 20I3 West Pacific Surveill response J WPSAR 20I6;7(3):I-5.

I2 Larson H, Hartigan-Go K, de Figueiredo A. Vaccine confidence plummets in the Philippines following dengue vaccine scare: why it matters to pandemic preparedness. Hum Vaccines Immunother 2019;15(3):630

I3 World Health Organization. Measles [Internet]. Vaccine-Preventable Diseases: Surveillance standards Geneva; 20I8. Available from: https://apps.who.int/iris/handle/10665/275754

I4 StataCorp. In: Stata Statistical Software: Release I6.I, College Station, TX: StataCorp LP; 2020.

I5 QGIS Geographic Information System (3.14). Open Source Geospatial Foundation Project. http://qgis.osgeo.org.

I6 Qin S, Ding Y, Yan R, He H. Measles in Zhejiang, China, 200420I7: Population Density and Proportion of Floating Populations Effects on Measles Epidemic. Heal Secur 20I9;I7(3).

I7 Philippines Statistics Authority. Population of the National Capital Region (Based on the 2015 Census of Population) [Internet]. [cited 2020 Feb 7]. Available from: https://psa.gov.ph/content/population-national-capital-region-based-2OI5-census-population-o

I8 Ducusin MJU, de Quiroz-Castro M, Roesel S, Garcia LC, CecilioElfa D, Schluter WW, et al. Using the World Health Organization Measles Programmatic Risk Assessment Tool for Monitoring of Supplemental Immunization Activities in the Philippines. Risk Anal 20I7;37(6):I082-95.

I9 Cilla G, Vicente D, Montes M. Robustness of measles immunity in parturient women in Gipuzkoa, Basque Country, Spain, in the postvaccination era. Eurosurveillence 2007: 7-I3.

20 Martins CL, Garly ML, Balé C, Rodrigues A, Ravn H, Whittle HC, et al. Protective efficacy of standard Edmonston-Zagreb measles vaccination in infants aged 4.5 months: Interim analysis of a randomised clinical trial. BMJ 2008;337(7665):339-43. 
2I Lo Vecchio A, Krzysztofiak A, Montagnani C, Valentini P, Rossi N, Garazzino S, et al. Complications and risk factors for severe outcome in children with measles. Arch Dis Child 2020;I05(9):896-9.

22 Paules C, Marston H, Fauci A. Measles in 20I9 - Going Backward. $N$ Engl J Med 2019: 2185-7.

23 Science M, Savage R, Severini A, McLachlan E, Hughes SL, Arnold $\mathrm{C}$, et al. Measles antibody levels in young infants. Pediatrics 20I9;I44(6).

24 Lee C, Hagan J, Jantsansengee B, Altanchimeg S, Yadamsuren B, Tserendorj C, et al. Increase in Infant Measles Deaths During a Nationwide Measles Outbreak-Mongolia, 20I5-20I6. J Infect Dis 20I0;220(II):I77I-9.

25 Donadel M, Stanescu A, Pistol A, Stewart B, Butu C, Jankovic D, et al. Risk factors for measles deaths among children during a Nationwide measles outbreak - Romania, 20I6-20I8. BMC Infect Dis 2O2I;2I(I):I-IO.

26 Imdad A, Mayo-Wilson E, Herzer K, Bhutta ZA. Vitamin A supplementation for preventing morbidity and mortality in children from six months to five years of age. Cochrane Database Syst Rev 20I7;20I7(3)
27 D'Souza R, D, Souza R. Vitamin A for treating measles in children. Cochrane Database Syst Rev 2005

28 Choe YJ, Hu JK, Song KM, Cho H, Yoon HS, Kim ST, et al. Evaluation of an expanded case definition for vaccine-modified measles in a school outbreak in South Korea in 20I0. Jpn J Infect Dis 20I2;65 (5):37I-5.

29 Fatima K, Syed NI. Dengvaxia controversy: Impact on vaccine hesitancy. J Glob Health 20I8;8(2):8-10.

30 Melenotte C, Zandotti C, Gautret P, Parola P, Raoult D. Measles: is a new vaccine approach needed? Lancet Infect Dis [Internet] 2018;18 (I0):I060-I. https://doi.org/IO.IoI6/SI473-3099(I8)30543-7.

3I World Health Organization. Measles and rubella strategic framework 202I-2030 [Internet]. Geneva; 2020. Available from: https:// s3.amazonaws.com/wp-agility2/measles/wp-content/uploads / 2020/II/measles_rubella_initiative_final_print.pdf

32 Hutchins SS, Papania MJ, Amler R, Maes EF, Grabowsky M, Bromberg $\mathrm{K}$, et al. Evaluation of the measles clinical case definition. $J$ Infect Dis 2004;I89(SUPPL. I) 